\title{
El orden en prisión: ¿solo una cuestión de seguridad? A propósito del caso de las prisiones de mujeres
}

\author{
Ares Batlle Manonelles \\ Universitat Pompeu Fabra \\ ares.batlle@upf.edu
}

\section{Resumen}

Este texto pertenece a una mesa redonda que ofrece un debate con James B. Jacobs, autor del clásico de la sociología de las prisiones Stateville: The penitentiary in mass society, sobre la vigencia de su obra cuarenta años después. Este comentario se ocupa de la cuestión del equilibrio entre orden y seguridad en las prisiones. Centrándonos en la realidad de las prisiones de mujeres, se reflexiona sobre la importancia del orden en las cárceles, cuál es su relación con la seguridad y cómo afecta a los objetivos rehabilitadores de la prisión. Concretamente, se piensa sobre la presencia que adquieren el orden y el control en las prisiones de mujeres, aun cuando la amenaza a la seguridad no es especialmente elevada o inminente.

Palabras clave: orden; seguridad; control; prisión; género

Abstract. Orden in prison: just a matter of security? Focus on women's prisons

This text belongs to a round table which offers a discussion with James B. Jacobs, author of the classic in sociology of prisons Stateville: The penitentiary in mass society, on the importance of his work 40 years later. This commentary considers the issue of balance between order and security in prisons. Focusing on women's prisons, we think about the importance of order in prison, which is the relationship between order and security and it affects the rehabilitation purposes of the prison. We reflect how order and control are developed in women's, which role order takes, even when there is not a big threat to security.

Key words: order; security; control; prisons; gender 


\title{
Sumario
}

\author{
1. El orden en Stateville \\ 2. La relación entre seguridad y orden. \\ Planteamiento del debate \\ 3. El orden en prisión: reflexión sobre \\ las prisiones de mujeres \\ Conclusión
}

Referencias bibliográficas

Un tema central en Stateville, que también ha recibido una amplia atención por parte de la literatura criminológica, es el orden en prisión. Los estudios clásicos sobre orden en prisión se han dirigido principalmente en dos sentidos. Por un lado, algunos trabajos intentan explicar cómo se impone o se mantiene el orden en prisión, intentando comprender qué elementos son relevantes para mantener el orden y qué papel desempeña el propio orden en la organización de la prisión (algunos de los principales autores de estas aportaciones son Bottoms y Sparks o Goffman). Por otro lado, varios estudios pretenden explicar el orden social de la prisión, es decir, qué tipo(s) de organización social encontramos en prisión y los elementos que la configuran, prestando especial atención a las relaciones y dinámicas sociales entre las personas presas, aquello que en la literatura criminológica se conoce como inmate society (véase, por ejemplo, Irwin, 1970 o Sykes, 1958). Estas aproximaciones no son incompatibles entre sí y varias investigaciones, como es el caso de Stateville, abordan el estudio del orden en prisión desde ambas perspectivas. En el presente trabajo, no obstante, nos ocuparemos solamente de la primera acepción, esto es, del orden en prisión entendido en términos de control y seguridad.

Como el mismo Jacobs advierte en su respuesta presentada durante el seminario, definir el concepto de orden no es sencillo, pues las contribuciones clásicas de la sociología del castigo lo han definido de formas diversas (Jacobs, 2018; Sparks, Bottoms y Hay, 1996). Algunos autores conceptualizan el orden en prisión como "la ausencia del desorden». Por ejemplo, Giddens entiende el orden como «sinónimo relativo de patrones o ausencia de caos» (1976: 98), y Young como «un patrón de relaciones que forman un conjunto» (1987:106), lo cual implica un elemento de continuidad y durabilidad a lo largo del tiempo. Por su parte, Sparks, Bottoms y Hay (1996) adoptan una definición más completa y definen el orden como el rango de prácticas y actividades que devienen rutinarias y asentadas en la vida cotidiana de la institución. Por otro lado, reservan el término control para las estrategias usadas para mantener un régimen considerado «ordenado». De manera similar, Young también define control como los mecanismos para la creación y el mantenimiento de dichos patrones.

Así pues, en este trabajo se utiliza el concepto de orden entendido como régimen (incluyendo el régimen penitenciario oficial y el informal), esto es, todas aquellas normas y rutinas que conforman la estructura, la organización y la cotidianidad de las prisiones, y que además son percibidas y defendidas como el buen funcionamiento de la institución. 
En este trabajo no nos concierne cómo se mantiene el orden sino su importancia y presencia en la vida penitenciaria y su relación/rol con la seguridad y el control y su presencia, especialmente centrándonos en las prisiones de mujeres. Concretamente, nos interesa reflexionar sobre si el orden en prisión está dirigido a asegurar el buen funcionamiento de la prisión en términos de seguridad ${ }^{1}$ o si ha tenido otras finalidades y cuáles. En el caso de las prisiones de mujeres, este aspecto ha sido puesto de manifiesto de manera especial, dado que dentro del sistema carcelario suponen instituciones con bajo riesgo en términos de seguridad y, por lo tanto, se ha cuestionado el uso de ciertas normas o regímenes disciplinarios estrictos (Carlen y Worrall, 2004; Kruttschnitt, 2011). Por ello, a continuación se expone cómo se desarrolla la cuestión del orden en prisión en Stateville y, más adelante, se plantean algunas reflexiones en torno al orden en las prisiones de mujeres.

\section{El orden en Stateville}

Sobre el orden en Stateville, nos hemos querido centrar en aquellas partes que nos hicieron surgir las preguntas aquí planteadas. Para ello, nos fijamos en la descripción que hace Jacobs del régimen autoritario, principalmente representado por el director Ragen en su mandato desde 1936 hasta 1962. Ragen dirigió la prisión con un liderazgo autoritario y de dominación sustentado en su alto carisma, prestigio y poder personal. El régimen de Ragen era absolutamente totalitario y en la gestión penitenciaria tenía gran importancia la autoridad y el mantenimiento del orden, dado que se entendía que cualquier desafío a la autoridad podía llevar a la rebelión. De hecho, «no estaba dispuesto a aceptar la menor oposición, ni siquiera a las normas insignificantes» (Jacobs, 1977: 202).

Un sistema tan autoritario como el de Ragen conlleva una sobrerregulación de la rutina y dominación de la vida en la prisión, especialmente para los internos pero también para los trabajadores. Además, aquellos que llamaban la atención por osar desafiar el sistema recibían largas y duras sanciones, como el aislamiento por tiempo indeterminado. Sin embargo, el sistema de disciplina era tan estricto que solo podía funcionar haciendo innumerables excepciones, convirtiendo el sistema en arbitrario (según favoritismos del personal, la indispensabilidad de algunos internos o el intercambio de informaciones). Existía coerción arbitraria junto a una constante e implacable supervisión y un sistema de «recompensas» para garantizar el mantenimiento del orden y el control.

A partir de los años cincuenta, con el auge del modelo rehabilitador, Ragen se ve obligado a justificar y adaptar su sistema autoritario de control en los términos de legitimidad y rehabilitación que estaban imponiendo. Así pues, se genera el discurso de que a «través de la obediencia de las normas, los internos serían resocializados» (Jacobs, 1977: 46). La intransigencia del régimen peni-

1. Entendiendo la seguridad en prisión en un sentido amplio (custodia de los internos, ausencia de violencia, infracciones disciplinarias, etc.). 
tenciario conseguiría llevar a los internos hacia la conformidad y la obediencia, que en última instancia les haría respetar las normas y la ley.

\section{La relación entre seguridad y orden. Planteamiento del debate}

Concretamente la pregunta planteada al profesor Jacobs en el seminario se basa en la siguiente afirmación: «El tipo de restricciones que se usaban sobre la libertad de los internos que se imponían en nombre de la seguridad a menudo parecían estar lejos de ser respuestas a amenazas inminentes a la seguridad» (p. 41). Este planteamiento ha llevado a querer dirigir la presente reflexión hacia el balance entre la seguridad y el orden y su rol en la organización penitenciaria. A menudo vemos que en las prisiones se implementan regímenes (sistemas de orden) con altos niveles de control y disciplina bajo la justificación de que son necesarios para mantener la seguridad. Pero, incluso en los casos donde no se precisarían regímenes de control y disciplina tan estrictos, dado que la amenaza a la seguridad no es grave ni inminente, encontramos regímenes con niveles de exigencia sobre el orden y la disciplina más elevados de los necesarios para mantener la seguridad. Además, ello suele resultar en altos niveles de sanciones por conductas menores, como ocurre en las prisiones de mujeres en nuestro contexto ${ }^{2}$. En este sentido, nos cuestionamos si en los sistemas penitenciarios a veces se usan el control, la disciplina o la autoridad para asegurar el orden incluso cuando se presentan como mecanismos para garantizar la seguridad.

A este planteamiento debemos aportar dos reflexiones previas que nos ofreció el mismo autor (Jacobs, 2018). En primer lugar, respecto a la relación entre orden y seguridad, debemos considerar que es una cuestión para reflexionar sobre la sociedad en general, como muchos autores han estudiado y mostrado. Esta tensión la encontramos en otros ámbitos, como, por ejemplo, en las medidas para la lucha contra el terrorismo o el debate sobre el uso y abuso de las identificaciones policiales. En segundo lugar, antes de abordar algunas reflexiones sobre las preguntas planteadas, cabe aclarar que el sistema de Ragen se basaba en el terror y las reglas arbitrarias. No era un sistema con un reglamento establecido, sino que se hacían las normas a medida y, por lo tanto, no había respeto al principio de legalidad ni garantías procesales. Así, se llegaron a usar medidas extremas y violentas contra los internos, lo que generó un sistema absolutamente basado en el control y la amenaza de violencia. Por supuesto, dicha realidad no se corresponde con la nuestra y, aunque la reflexión surge de ella, no se pretende establecer paralelismos.

\section{El orden en prisión: reflexión sobre las prisiones de mujeres}

Como consideración previa, cabe recordar que, en términos generales, una de las principales finalidades de las prisiones es la custodia de las personas presas; así lo define la propia Ley Orgánica 1/1979, de 26 de septiembre,

2. Datos disponibles a través del portal de trasparencia de la Generalidad de Cataluña. 
General Penitenciaria. En este sentido, se podría argumentar fácilmente que garantizar la seguridad en las prisiones en gran parte implica asegurar con éxito dicha custodia de las personas presas. Sobre la base de este supuesto, autoras como Gartner y Kruttschnitt (2004), en su análisis de la evolución del sistema penitenciario estadounidense, resaltan que la custodia siempre será la base del sistema penitenciario, y el énfasis que se ponga en la rehabilitación o en un sistema más gerencial siempre será sobre la base del sistema de custodia, nunca en sustitución de este. Por ello no resulta difícil comprender que, aun en un sistema penitenciario como el español, donde claramente se establece la finalidad rehabilitadora de la pena de prisión, la seguridad sigue teniendo un elevada presencia e importancia (Cutiño, 2015), tanto en la organización de los centros como en la toma de decisiones o en la gestión de recursos (véase, por ejemplo, la distribución del personal penitenciario según el ámbito de intervención en la SGIP, 2014).

Cabe tener presente que todas las prisiones tienen altos niveles de control situacional comparado con otros contextos vitales (Sparks, Bottoms y Hay, 1996). Como expuso Goffman (1961), las instituciones totales generalmente hacen valer su autoridad con un nivel de detalle cualitativamente superior a otros contextos de vida. Así pues, como Foucault (1976) argumenta, las estrategias de control van más allá del régimen disciplinario en sí. En este sentido, nos referimos al uso del trabajo remunerado en prisión como incentivo y al miedo a no obtener un permiso o la condicional como amenaza. Se destaca como mecanismo de control fundamental la rutina y el control del tiempo (Bottoms, 1999). De este modo, la autoridad se refuerza con un abanico de recompensas y sanciones extensivo y detallado, de nuevo teniendo presente que, en la práctica, responde a infracciones o desajustes considerados demasiado triviales para merecer atención en la vida fuera de prisión (Goffman, 1961). Además, actualmente estos mecanismos de control se mezclan con las prácticas más modernas de gestión gerencial penitenciaria reforzadas por las actuales corrientes de gestión del riesgo (Sparks, Bottoms y Hay, 1996). Como se ha mencionado, es especialmente importante plantear estas cuestiones en el caso de las prisiones de mujeres por ser, en general, prisiones de bajo riesgo en términos de seguridad.

Además, para reflexionar sobre qué papel desempeñan el orden y la seguridad en la institución penitenciaria, deben considerase dos razonamientos en relación con la finalidad rehabilitadora de las prisiones.

En primer lugar, podríamos entender el orden y la seguridad como elementos básicos para que exista trabajo rehabilitador, ya que sin un mínimo habría demasiada inseguridad y violencia y no se podría hacer ningún trabajo ni programa dirigido a la rehabilitación (DiIulio, 1987). Pero, a veces, parece que el orden pasa a ser una finalidad en sí mismo, es decir, nos olvidamos de que el orden por sí solo no es el objetivo. En estos casos se tiende a la sobrerregulación y al abuso de las normas disciplinarias y cuando el remedio puede ser peor que la enfermedad (Irwin, 2005). Si analizamos el elevado número de infracciones menores presentes en las prisiones de mujeres por actos como 
manchar las sábanas o no hacer bien la cola para comer, nos deberíamos preguntar si estas sanciones realmente responden a la finalidad de garantizar la seguridad. Históricamente se ha evidenciado que en las cárceles de mujeres las amenazas a la seguridad son mínimas (Bosworth, 1996). Por ejemplo, en los últimos quince años no ha habido ninguna fuga de mujeres presas en las cárceles catalanas y solo dos tentativas ${ }^{3}$. Sin embargo, en el estudio exploratorio de Giménez-Salinas y colaboradores (2006) se observa que alrededor del $60 \%$ de las mujeres presas en Cataluña se sienten permanentemente controladas por el personal de vigilancia.

En segundo lugar, podríamos pensar en el orden y la seguridad como mecanismos rehabilitadores (como se menciona en Stateville). Es decir, a través de enseñarles a seguir un orden, les enseñamos a poder convivir con normas, como deberán hacer en el exterior. Aunque ello podría parecer lógico y adecuado, debemos considerar algunos posibles peligros adyacentes. Por un lado, podemos estar enseñando conformidad y sumisión sin cometido rehabilitador (Goffman, 1961), como se ha hecho en especial en las cárceles de mujeres históricamente (Almeda, 2005). Además, en estos casos, la rehabilitación exige conformidad y asunción de los estándares impuestos por el régimen penitenciario, con los importantes y limitantes sesgos de género que este puede conllevar (Almeda y Bodelón, 2007, sobre domesticidad en las cárceles de mujeres). Más aún, podemos caer en la supresión de la agencia de las personas presas, es decir, en lugar de dotar a las internas con herramientas de autonomía y empoderamiento para su salida, las estamos «inhabilitando», ya que solo serán capaces de funcionar en un entorno hiperregulado y con poco espacio para la autogobernabilidad (véase Hannah-Moffat, 2001 y McCorkel, 2013).

\section{Conclusión}

En esta pequeña reflexión se pretendía pensar sobre cuál es la finalidad y el papel del orden en prisión. Especialmente cuestionar su aparentemente inquebrantable relación con la seguridad y, por lo tanto, invitar a considerar el uso que adquiere el orden, fundamentalmente en contextos donde parece que la seguridad no está altamente amenazada. Las prisiones de mujeres son instituciones en su mayoría más seguras que las prisiones masculinas, donde los niveles de violencia explícita o violencia física (especialmente hacia los funcionarios) son menores y donde el nivel de complacencia con las normas y el régimen suele ser bastante elevado (en parte, por la propia reproducción de los roles de género femeninos). Así pues, parece necesario cuestionar principalmente dos aspectos. Por un lado, si estamos haciendo "demasiados esfuerzos» (demasiadas normas y mecanismos de control) para garantizar una seguridad que, hasta ciertos niveles, existe en general en las prisiones de mujeres. En cualquier caso, debemos plantearnos si sería más fácil y adecuado garantizar mayor seguridad a través de otros sistemas menos autoritarios e invasivos. Por otro lado, como

3. Dato obtenido a través del portal de trasparencia de la Generalidad de Cataluña. 
consecuencia de lo anterior, plantear la necesidad de la elevada penalización de comportamientos menores o la elevada exigencia de cumplir con regulaciones «al minuto» del día a día de las personas presas, no justificado ni por razones de seguridad ni por finalidades resocializadoras 4 .

Los sistemas penitenciarios que priorizan la custodia y la seguridad usualmente adquieren regímenes disciplinarios y gerenciales más estrictos (Gartner y Kruttschnitt, 2004). Sin embargo, como hemos mencionado, el uso extensivo del control puede empobrecer los otros objetivos que la prisión tiene encomendados (Sparks, Bottoms y Hay, 1996). Aunque el avance en nuestros sistemas penitenciarios es innegable en muchos sentidos (Cid, 2002), ello no implica que debamos dejar de poner en tela de juicio aspectos que quizá se hayan naturalizado en los nuevos modelos de gestión penitenciaria.

\section{Referencias bibliográficas}

AlmEDA, Elisabet (2005). «Pasado y presente de las cárceles femeninas en España». Sociológica, 6, 75-106.

AlmEDA, Elisabet y BODElón, Encarna (2007). Mujeres y castigo: un enfoque sociojurídico y de género. Oñati: Instituto Internacional de Sociología Jurídica de Oñati.

BOSWORTH, Mary (1996). «Resistance and Compliance in Women's Prisons: Towards a Critique of Legitimacy». Critical Criminology, 7 (2), 5-19. <https://doi.org/10.1007/BF02461111>

ВоттомS, Anthony (1999). «Interpersonal Violence and Social Order in Prisons». Crime and justice, 26, 205-281. <https://www.jstor.org/stable/1147687>

CARLEn, Pat y Worrall, Anne (2004). Analysing Women's Imprisonment. Londres: Willan Publishing.

CiD, José (2002). «El sistema penitenciario en España». Jueces para la democracia, 45, $15-27$.

CutiÑo, Salvador (2015). «Algunos datos sobre la realidad del tratamiento en las prisiones españolas». Revista Electrónica de Ciencia Penal y Criminologia, 17, 1-41.

DiIULIO, John (1987). Governing Prisons. Nueva York: The Free Press.

FOUCAUlT, Michel (1976). Vigilar y castigar: nacimiento de la prisión. México: Siglo XXI.

GARTNER, Rosemary y KRUTTSCHNITT, Candace (2004). «A Brief History of Doing Time: The California Institution for Women in the 1960s and the 1990s». Law \& Society Review, 38 (2), 267-304. <https://doi.org/10.1111/j.0023-9216.2004.03802009.x>

GidDENS, Anthony (1976). New Rules of Sociological Method. Londres: Hutchinson.

GimÉneZ-Salinas, Esther; Riera, Jordi; Botella, Lluís y Marteache, Nerea (2006). «L'opinió de les dones recluses a Catalunya sobre l'encarcerament». Justidata, 45, 1-16.

GOFFMAN, Erving (1961). «On the characteristics of total institutions». En: Donald Cressey y Johan Galtung (eds.). The Prison: Studies in Institutional Organization and Change. Nueva York: Holt, Rinehart and Winston.

4. Con ello no queremos decir que un argumento similar no se podría plantear para las prisiones en general, simplemente nos centramos en las prisiones de mujeres. 
Hannah-Moffat (2001). Punishment in Disguise. Toronto: University of Toronto Press. <https://doi.org/10.3138/9781442678903>

IRWIN, John (1970). The Felon. Englewood Cliffs: Prentice-Hall Inc.

- (2005). The Warehouse Prison: Disposal of the New Dangerous Class. Los Angeles: Roxbury.

JACOBS, James (1977). Stateville: The penitentiary in mass society. Chicago: University of Chicago Press.

- (2018). «Author meets critics: Stateville Revisited», III Conferencia Internacional del Máster en Criminología y Ejecución Penal. Barcelona, 11 de mayo.

KRUTTSChnitT, Candace (2011). «Women's Prisons». En: Michael Tonry (ed.). The Oxford Handbook of Crime and Criminal Justice. Nueva York: Oxford University Press.

Ley orgánica 1/1979, de 26 de septiembre, general penitenciaria.

MCCORKEL, Jill (2013). Breaking Women. Gender, Race, and New Politics of Imprisonment. Nueva York y Londres: New York University Press.

SGIP (Secretaría General de Instituciones Penitenciarias) (2014). El sistema penitenciario español. Madrid: Ministerio del Interior.

Sparks, Richard; BotToms, Anthony y Hay, Will (1996). Prison and the Problem of Order. Oxford: Clarendon Press. <https://doi.org/10.1093/acprof:oso/9780198258186.001.0001>

SYKES, Gresham (1958). The Society of Captives: a study of a maximum security prison. Princeton: Princeton University Press.

YounG, Peter (1987). «The concept of social control and its relevance to the prison debate». En: Anthony Bottoms y Roy Light (eds.). Problems of Long-Term Imprisonment. Aldershot: Gower. 